\title{
The COVID-19 Outbreak in Iran; The First Patient with a Definite Diagnosis
} \author{
$\mathrm{MD}^{3}$; Alireza Koohpaei, $\mathrm{PhD}^{6}$; Mohammad Abbasinia, $\mathrm{PhD}^{7}$, Ehsan Sharifipour, $\mathrm{MD}^{4 *}$; Samad EJ Golzari, $\mathbf{M D}^{8}$

\begin{abstract}
'Gastroenterology and Hepatology Diseases Research Center, Qom University of Medical Sciences, Qom, Iran
${ }^{2}$ Department of Public Health, Faculty of Health, Qom University of Medical Sciences, Qom, Iran

${ }^{3}$ Department of Infectious Diseases, Faculty of Medicine, Qom University of Medical Sciences, Qom, Iran

${ }^{4}$ Neuroscience Research Center, Qom University of Medical Sciences, Qom, Iran

${ }^{5}$ Cellular and Molecular Research Center, Qom University of Medical Sciences, Qom, Iran

${ }^{6}$ Research Center for Environmental Pollutants, Qom University of Medical Sciences, Qom, Iran

'Department of Nursing, Faculty of Nursing and Midwifery, Qom University of Medical Sciences, Qom, Iran
\end{abstract} \\ ${ }^{8}$ Department of Anesthesiology and Intensive Care Medicine, Tabriz University of Medical Sciences, Tabriz, Iran
}

Mohammad Reza Ghadir, MD'; Ali Ebrazeh, MD²; Javad Khodadadi, MD³ Masumeh Zamanlu, MD'; Saeed Shams, MD; Maryam Nasiri,

\begin{abstract}
The novel coronavirus, formerly named as 2019 novel coronavirus (2019-nCov) caused a rapidly spreading epidemic of severe acute respiratory syndrome (SARS) in Wuhan, China and thereafter, progressed globally to form a pandemic of coronavirus disease 2019 (COVID-19) in numerous countries; and now confirmed cases are reported from several provinces of Iran. Now various medical centers, clinicians and researchers around the world share their data and experiences about COVID-19 in order to participate in the global attempt of controlling the pandemic. The current report investigates the clinical presentations and paraclinical findings of the first confirmed cases and mortalities in the initiation of the outbreak of COVID-19 in Iran. Cite this article as: Ghadir MR, Ebrazeh A, Khodadadi J, Zamanlu M, Shams S, Nasiri M, et al. The COVID-19 outbreak in Iran; the first patient with a definite diagnosis. Arch Iran Med. 2020;23(7):503-504. doi: 10.34172/aim.2020.48.
\end{abstract}

Received: April 6, 2020, Accepted: April 6, 2020, ePublished: July 1, 2020

\section{Introduction}

The severe acute respiratory syndrome coronavirus 2 (SARS-CoV-2) caused a rapidly spreading epidemic of severe acute respiratory syndrome (SARS) in Wuhan, China and thereafter progressed globally to form a pandemic of coronavirus disease 2019 (COVID-19) in numerous countries. ${ }^{1-3}$ Confirmed cases have been reported from several provinces of Iran. Currently, various medical centers, clinicians and researchers around the world share their data and experiences about COVID-19 in order to participate in the global attempt to control the pandemic. ${ }^{4}$ The current report investigates the clinical presentations and paraclinical findings of the first confirmed case and mortality in the onset of the COVID-19 outbreak in Iran.

\section{Case Report}

On February 7, 2020, a 75-year-old man with acute dyspnea and history of fever and nonproductive coughs since February 1 presented to our emergency department in Qom, Iran. He had neither a significant past medical history nor any recent travel or exposure to individuals with respiratory symptoms. However, a detailed history taken from his family revealed that he had repeatedly visited the Qom Bazaar (a large and traditional shopping center with numerous shops and crowded population) last week for shopping.

On clinical examination, he had a maximum temperature of $38.0^{\circ} \mathrm{C}$, respiratory rate of 24 breaths per minute and oxygen saturation of $85 \%$ on room air with respiratory distress and bilateral pulmonary rales. Chest X-ray and chest CT scan (Figure 1) revealed bilateral lung involvement. Laboratory investigations showed decreased lymphocyte percentage (13\%) with marginally decreased lymphocyte count (1053 cells per $\mathrm{mm}^{3}$ ), increased neutrophil percentage (85\%), yet normal neutrophil $\left(6885\right.$ cells per $\left.\mathrm{mm}^{3}\right)$ and total white blood cells $\left(8100\right.$ cells per $\mathrm{mm}^{3}$ ), ESR 90 seconds, yet negative CRP, creatinine concentration $1.1 \mathrm{mg} / \mathrm{dL}$, BUN $31 \mathrm{mg} /$ $\mathrm{dL}$, and near normal liver function tests. During three days of hospitalization, despite administration of empiric antibiotics (azithromycin, vancomycin, meropenem and oseltamivir) and supportive measures, his respiratory symptoms were aggravated. Nevertheless, oxygen saturation stayed $>93 \%$ with oxygen mask with reservoir bag. On the third day of admission, his creatinine and BUN levels increased $(\mathrm{Cr}=1.6 \mathrm{mg} / \mathrm{dL}$ and $\mathrm{BUN}=64$

*Corresponding Author: Ehsan Sharifipour, MD; Deputy of research and technology, Department of Neurology, Neurosciences Research Center (NSRC), Qom University of Medical Sciences, Shahid Beheshti Hospital, Shahid Beheshti St., Qom, Iran. Tel: +98-25-36122436; Fax: +98-25-36122946;

Email: Sharifipour.e@gmail.com 


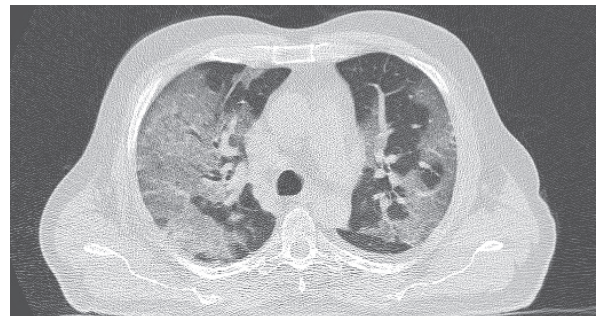

Figure 1. The First COVID-19 Case in Iran. Chest CT scan shows bilateral multiple patchy ground glass opacity with broncho-alveolar pattern.

$\mathrm{mg} / \mathrm{dL}$ ) and EKG (Figure 2) indicated right heart strain. Nasopharyngeal (mid-turbinate and throat) swabs obtained on admission were negative for influenza virus A and B, parainfluenza, human metapneumovirus, adenovirus, and respiratory syncytial virus. Reverse-transcriptase polymerase chain reaction (RT-PCR) assay was positive for 2019-nCoV on February 10 which was later confirmed through sequencing on February 19. On the fourth day of hospitalization, despite being conscious and afebrile, the clinical condition of the patient deteriorated suddenly over a few hours, necessitating intubation and mechanical ventilation. This was followed by progressive and resistant hypoxia and bradycardia; ultimately, was expired despite all the supportive therapy he was receiving.

Our case is believed to be the first COVID-19 case with a definite diagnosis in the disease outbreak in Iran (but not probably the first case in Qom or Iran, due to the high percentage of patients with mild symptoms of this disease). The clinical picture of our patient (i.e. initial fever, cough, bilateral pneumonia dominantly in the lower segments and lymphopenia) is similar to that of most hitherto described cases. Nevertheless, we believe that special consideration should be devoted to the cardiac involvement of these patients as in our case who developed cardiac dysrhythmia and ischemia and finally, cardiopulmonary arrest. Hence, thorough and vigilant cardiac monitoring is recommended in patients with either suspected or confirmed COVID-19.

\section{Authors' Contribution}

$\mathrm{MGH}, \mathrm{JK}, \mathrm{ESH}$, and $\mathrm{MN}$ had role in the diagnosis and treatment of this patient. $\mathrm{AE}, \mathrm{ESH}, \mathrm{MZ}, \mathrm{SSH}, \mathrm{AK}, \mathrm{MA}$ and $\mathrm{SG}$ were involved in providing clinical information and writing the case report manuscript.

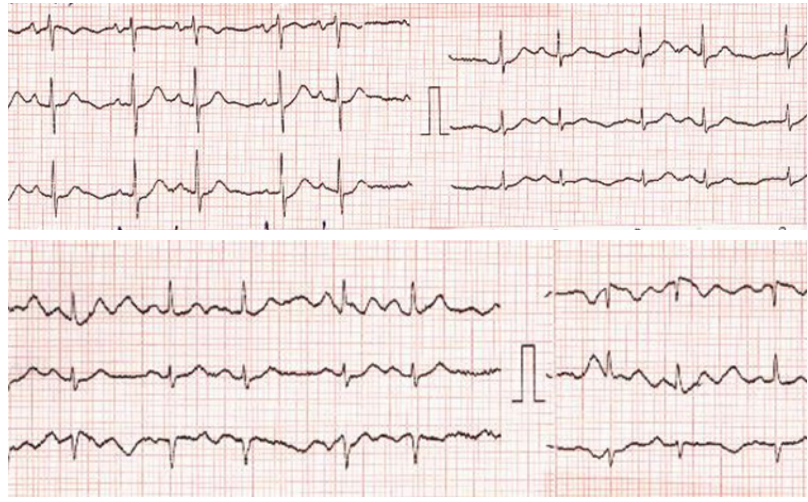

Figure 2. The First COVID-19 Case in Iran. EKG demonstrates heart rate of 100 beats per minutes, irregular rhythm, suspected p-pulmonale in the right chest leads and, upsloping ST-T depression while other EKG characteristics seem normal. The EKG findings could indicate heart strain specifically in the right chambers.

\section{Conflict of Interest Disclosures}

The authors have no conflicts of interest.

\section{Ethical Statement}

Informed consent was obtained from the patient's legal guardians for publication.

\section{Acknowledgments}

We wish to thank Naimeh Bozorgqomi, Tayebeh Sabokbar, Dr. Ava Mohammadzadeh Abachi, Dr. Farzad Moazzeni, and Dr. Azhar Eshraghi for useful efforts and comments.

\section{References}

1. Zu ZY, Jiang MD, Xu PP, Chen W, Ni QQ, Lu GM, Zhang LJ. Coronavirus Disease 2019 (COVID-19): A Perspective from China. Radiology. 2020:200490. doi: 10.1148/ radiol.2020200490.

2. Yang $Y$, Shang $W$, Rao X. Facing the COVID-19 outbreak: What should we know and what could we do? J Med Virol. 2020;92(6):536-7. doi: 10.1002/jmv.25720.

3. Lai CC, Shih TP, Ko WC, Tang HJ, Hsueh PR. Severe acute respiratory syndrome coronavirus 2 (SARS-CoV-2) and coronavirus disease-2019 (COVID-19): The epidemic and the challenges. Int J Antimicrob Agents. 2020;55(3):105924. doi: 10.1016/j.ijantimicag.2020.105924.

4. Chen X, Tian J, Li G, Li G. Initiation of a new infection control system for the COVID-19 outbreak. Lancet Infect Dis. 2020;20(4):397-8. doi: 10.1016/S1473-3099(20)30110-9. 